\title{
P9 Repeatability and Predictors of a Potentially Blood Pressure-Independent Parameter of Arterial Stiffness
}

\author{
Mark Butlin ${ }^{1}$, James Cox ${ }^{1}$, Bart Spronck ${ }^{2}$, Isabella Tan ${ }^{1}$, Alberto Avolio ${ }^{3, *}$ \\ ${ }^{1}$ Department of Biomedical Sciences, Faculty of Medicine and Health Sciences, Macquarie University, Sydney, Australia \\ ${ }^{2}$ School of Engineering and Applied Science, Yale University, New Haven, CT, USA \\ ${ }^{3}$ Macquarie University, Sydney, Australia
}

\section{ABSTRACT}

Background: Arterial stiffness (e.g., as measured by carotid-femoral pulse wave velocity (cfPWV)) changes acutely with blood pressure (BP). High cfPWV at high BP could be due to structural arterial changes or acute BP effects. Various parameters have been proposed as BP-independent parameters of arterial stiffness. These rely on assumptions to simplify theoretical calculations. This study evaluates a parameter quantifying BP dependency of cfPWV through BP manipulation, avoiding assumptions and mathematical derivations.

Methods: Brachial BP and cfPWV (SphygmoCor XCEL, AtCor Medical, Australia) were assessed in seated and supine position. The postural change invokes a systemic and hydrostatic BP change, thus changing cfPWV. $\Delta \mathrm{cfPWV} / \Delta \mathrm{BP}$ was calculated. Repeatability was assessed in 25 participants ( $28 \pm 11$ years (mean \pm SD), $52 \%$ female) across two visits on separate days. Crosssectional correlation with age, gender, height, weight, seated BP, and supine cfPWV was assessed by stepwise linear regression across 135 participants ( $49 \pm 23$ years, $48 \%$ female).

Results: Mean $\Delta \mathrm{cfPWV} / \Delta \mathrm{BP}$ was $0.061 \pm 0.025 \mathrm{~m} / \mathrm{s} / \mathrm{mmHg}$. Repeatability showed a between-measurement difference of $0.005 \pm 0.028 \mathrm{~m} / \mathrm{s} / \mathrm{mmHg}(8.2 \%$ error on mean). $\Delta \mathrm{cfPWV} / \Delta \mathrm{BP}$ was cross-sectionally correlated with seated diastolic BP (standardised $\beta=-0.506, p<0.001)$, systolic $\mathrm{BP}(\beta=0.503, p<0.001)$, weight $(\beta=-0.220, p=0.006)$, and heart rate $(\beta=0.178$, $p=0.039)$ (model $\left.p<0.05, r^{2}=0.264\right)$.

Conclusion: $\triangle \mathrm{cfPWV} / \triangle \mathrm{BP}$ showed fair repeatability. Notwithstanding accounting for BP, it correlated with typical long-term modifiers of arterial stiffness such as chronic elevated BP and weight. Further studies are required to validate if dividing $\Delta$ cfPWV by $\Delta \mathrm{BP}$, following acute intervention, creates an acceptable BP-independent arterial stiffness variable before the parameter is investigated for cardiovascular risk prediction to evaluate its clinical utility.

(c) 2019 Association for Research into Arterial Structure and Physiology. Publishing services by Atlantis Press International B.V. This is an open access article distributed under the CC BY-NC 4.0 license (http://creativecommons.org/licenses/by-nc/4.0/). 